\title{
The systems of the active rigidity and their application in transport machine
}

\author{
Aleksandr Kudryashov ${ }^{1, *}$, Ekaterina Igumensheva ${ }^{1}$, and Olesya Kopylova ${ }^{1}$ \\ ${ }^{1}$ Nosov Magnitogorsk State Technical University. Magnitogorsk, Russia
}

\begin{abstract}
The current state in the field of designing of mechanical systems is characterized by the application of an exhaustively complete set of traditional ways to increase rigidity, that contains the further growth of their principal specifications. Use of high-strength materials only aggravates a problem as their work is supposed at more high tensions, and, therefore, and larger sizes of elastic deformations. At the same time the modern breakthrough technologies in the field of automatic control allow to solve problems, the bound to a slackness of mechanical systems means of operating control of geometrical deviations and fluctuations, and their fissile suppression.
\end{abstract}

\section{Introduction}

The rigidity is an ability of structural elements to resist deformation at external influence. The systems of the fissile rigidity are understood as the fissile systems compensating such deformations.

The current state in the field of designing of mechanical systems is characterized by the application of an exhaustively complete set of traditional ways to increase rigidity, that contains the further growth of their principal specifications (load-carrying capacity coefficient, upkeep zone size, accuracy of positioning, amplitude-frequency characteristic).

Besides, the oscillating phenomena caused by a slackness of a mechanical system lead to emergence of alternate stresses in elements of designs that causes their aging, reducing terms of their service.

Use of high-strength materials only aggravates a problem, as their work is supposed at more high tensions, therefore, and larger sizes of elastic deformations.

At the same time the modern breakthrough technologies in the field of automatic control allow to solve problems, the bound to a slackness of mechanical systems means of operating control of geometrical deviations and fluctuations and their fissile suppression.

Having appeared in aircraft, space and difficult military equipment, similar technologies develop, improve, become quicker, cheaper and more reliably, what creates prerequisites to their more widespread introduction.

\footnotetext{
* Corresponding author: kudryashoffal@gmail.com
} 


\section{The state of studies in the area under consideration}

As analogs of the offered technology can be considered examples of use of similar technologies or solutions of similar problems.

For example, from 80th years of the last century "the fissile appendages" of racing cars in the class Formula One of team are known Lotus, and later, Williams. The essence of technology consisted in maintaining of a constant ground clearance.

By the beginning of the 90th this technology gave to the Williams team such a clear competitive advantage, that since 1994 it was forbidden by technical regulations.

Now the fissile appendages gain development in "civil" cars. They are in an arsenal of such auto makers as BMW (Dynamic Drive), Toyota (Kinetic Dynamic Suspension System, KDSS), Hyundai (Active Geometry Control Suspension, AGCS).

In an appendage Active Body Control, $\mathrm{ABC}$ от Mercedes-Benz the rigidity of a spring changes by means of a hydraulic pressure drive which provides forcing of oil in a damping rack under high pressure.

Control of hydraulic cylinders of damping racks is exercised by electronic system which turns on 13 various sensors (provisions of a body, longitudinal, transversal and vertical acceleration, pressure) the control unit and actuation mechanisms - magnet valves. The ABS system almost completely excludes body cranes under various traffic conditions (turn, acceleration, braking).

The pendant of Magic Body Control became further development of the $\mathrm{ABC}$ system, which watches a covering in real time and adjusts elements under a concrete situation.

The front-facing camera monitors a surface apart of 15 meters; from the front-facing camera sensors obtain information allowing changing characteristics of the damping elements for fractions of a second.

The similar system was presented by the Audi Company. The express camera scans the road of 18 times to second, electronic devices calculate as it is necessary to react that in salon nobody felt rough nesses.

Milliseconds are spent for calculations, and then at the right time the computer sends team to the executive electric motors stabilizers to draw in the necessary wheel over a hummock or a hole, and also to the damping elements: to electronically controlled shockabsorbers and pneumosprings.

Similar example is the operator manipulator which got the nickname "Russian Hand" in Hollywood. Established on a roof of the car, the manipulator compensates pushes from rough nesses of the road, providing the smoothly varying movement of the camera on the given by the operator of a trajectory. Such ability is provided by the gyrostabilizer head equipped with electronic sensors of a vertical. These sensors issue commands to the servodrivers holding the camera in the necessary direction.

"Damper", caused by wind, a skyscraper of Citigroup center (Citicorp-center) can be other example of application of the fissile suppression of deformations and fluctuations. In the skyscraper constructed in 1977 on a roof the 440-ton concrete block is installed. Connected to the computer, it is displaced in process of strengthening of wind and suppresses the fluctuations of all design caused by it.

Today for fight against a swing in an upper of high-rise buildings establish the huge counterbalances called tuned mass dampers. In most cases TMD shock-absorbers represent a huge sphere from steel or concrete which weight reaches 300-800 tons. This sphere is suspended on springs and pistons in an upper of buildings and balances at change of a slope angle of the building.

Thus, the systems of the fissile rigidity are efficiently operated already now. Today the task consists in development of scientific base for widespread introduction of such systems. 
Can be spheres of their application as manipulators (from metallurgical cranes manipulators to mild manipulators for work in space), and antenna radars, telescopes, machines.

\section{Principle of work of system of the fissile rigidity}

As an example we will consider such transport vehicle as the automobile hydraulic elevator (AHE). The demand of such cars constantly grows, and together with increase in number of storey of structures also requirements to height of rising of a cradle grow. The poor rigidity of a design in this case will entail a cradle swing with amplitudes exceeding norms of safe operation.

The systems of the fissile rigidity will fight in this case against geometrical deviations and a swing by means of their automatic control and compensation of deviations. They represent set of the mechanical system which is carrying out correctional impacts on executive body of the manipulator, a measuring system, making the continuous measurement of provision of an action (auto tower cradle) and/or geometries of links of executive body and control system forming on the basis of the obtained data of a measuring system pilot signals on drives of a mechanical system. The structure of such device is shown in the figure 1 .

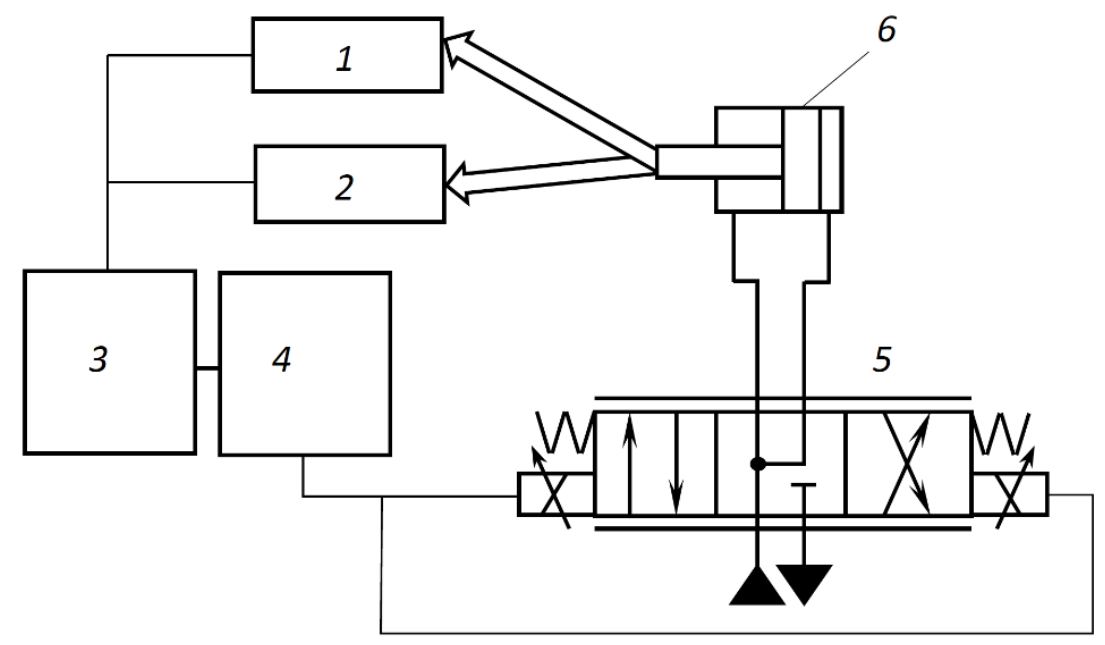

Fig. 1. Block diagram of the device of automatic control and compensation of fluctuations and geometrical deviations

The measuring system of the device represents the block 1 of accelerometers and gyroscopic sensors and strain-measuring system 2 , sending data respectively on the provision of a cradle and geometrical parameters of links of the manipulator in a control system incorporating the calculator 3 processing the obtained data by their comparison with the preset values and the forming pilot signals and the amplifier 4, transforming these signals to the currents entering on coils of proportional management of the choking hydraulic valve 5 , carrying out the dosed giving of a hydraulic medium in piston or rod end of a hydraulic cylinder 6 , the compensating cradle deviation.

One of simple options of realization of a mechanical system is the auto tower hydraulic system superstructure, connected to entrances «a» and «b» of the executive drives (figure 2). 


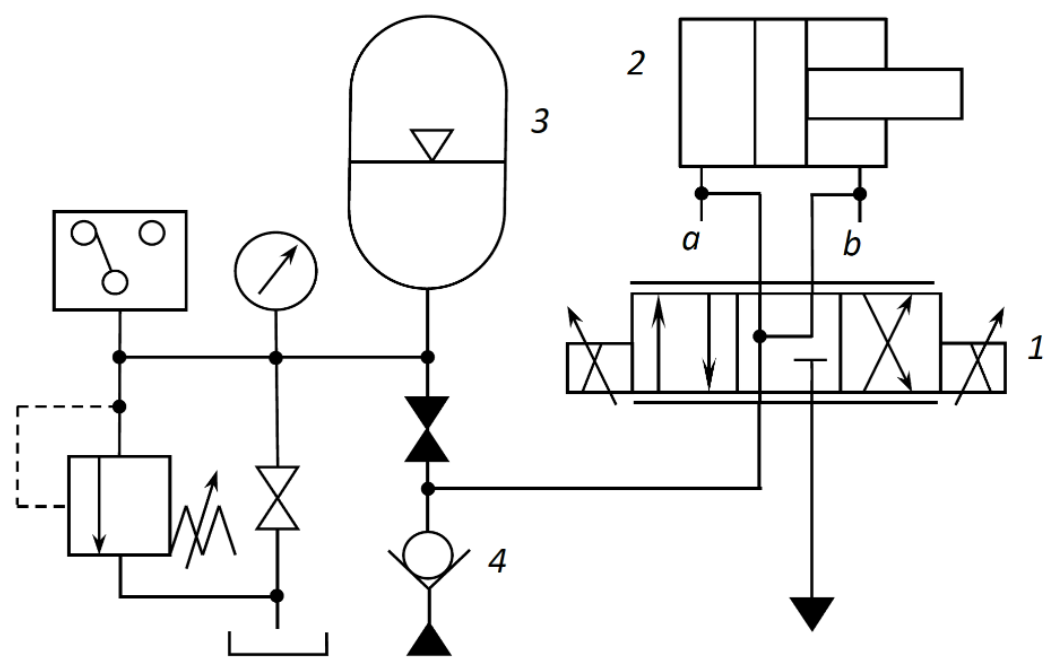

Fig. 2. The key diagram of a hydraulic actuator.

Feature of this scheme is that the distributor 1 with proportional management pressurizes in both cavities of a hydraulic cylinder 2, and change of force of currents on coils of management leads to the liquid dosed to giving/dumping in piston / rod cavities. Such scheme provides the maximal quick action. For minimization of delays of working off of teams the distributor is placed immediately at a hydraulic cylinder, and a source of pressure is the hydro accumulator 3 located right there. At the working hydraulic system, the hydro accumulator is constantly recharged via the backpressure valve 4 ; therefore requirements to its volume are minimal.

With such superstructures both all hydraulic cylinders and their part minimum necessary for adjustment in all coordinate directions can be equipped (for example, only hydraulic cylinders of portable support).

The measurement of provision of a cradle can be made by the block of accelerometers and gyroscopic sensors. Now such systems from the category of expensive devices of aircraft turned into structure of control systems of the budgetary unmanned aerial vehicles (drones). And, such systems can transfer information on wireless technology.

For monitoring of geometry of links of the manipulator the progressive systems of a strain measurement, including nanosheet can be used.

For system effectiveness of the fissile rigidity the quick action is crucial. Reaction rate is influenced by several factors including a rigidity of a mechanical system. So, response time on AHE cradle from the correcting impulse in inverse proportion to a natural frequency of fluctuations of that part of a mechanical system, which settles down between a cradle and a source of the correcting impulse. And the source will be farther from a cradle, the frequency and, respectively, longer response time will be lower. That is, if to compensate cradle deviations only by means of hydraulic cylinders of portable support, then the quick action can be poor. In this case for compensation of the delays caused by a slackness of links of a mechanical system the relative frame weight fixed on the cradle and moved according to a vector of speed of a deviation of the last can be used it is similar to the "damper of skyscrapers" described above (figure 3). 


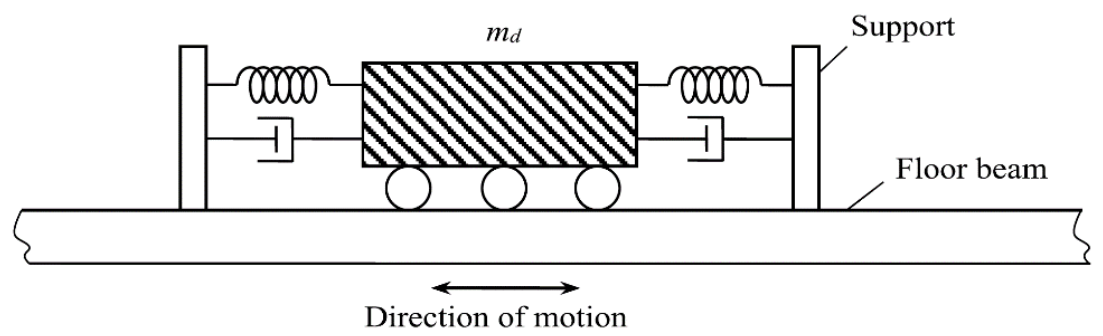

Fig. 3. Schematic diagram of a translational tuned mass damper

\section{Conclusions}

Now work on computer model operation of AHE, its dynamic analysis with assessment of effectiveness of options of realization of system of the fissile rigidity is conducted. Development of the equipment for AHE and its test on an actual exemplar which have to give an assessment of prospects of this development will be the following stage.

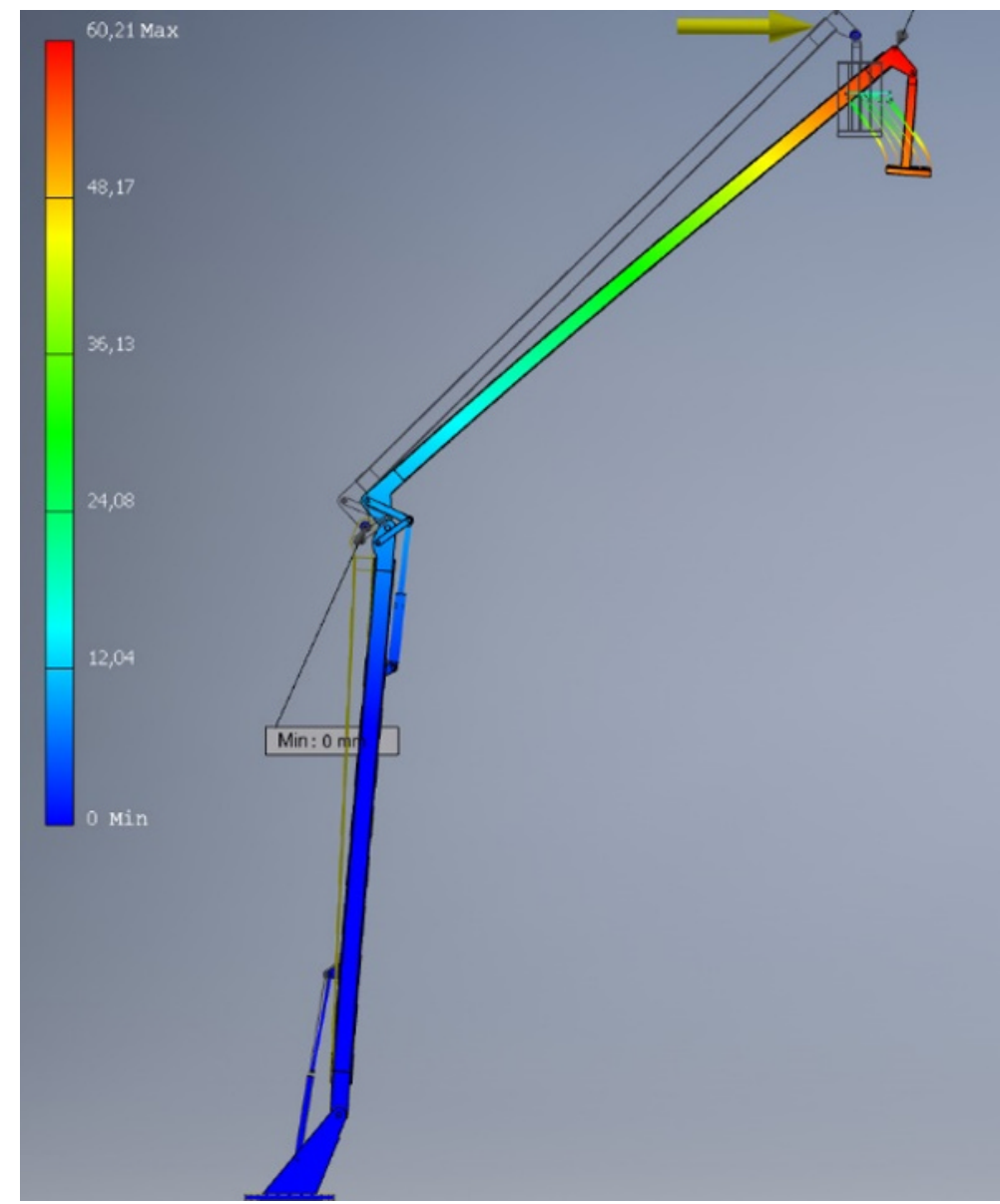

Fig. 4. Computer model operation of AHE 


\section{References}

1. "Active suspension". Motor Sport Magazine. Retrieved 2017-05-14. (December 2001)

2. "The new Mercedes-Benz S-Class - The aspiration: the best automobile in the world; Suspension: The world's first suspension system with "eyes"" (Press release). Daimler AG. (15 May 2013). Retrieved 7 (June 2013)

3. "Looking ahead to the new Audi A8: Fully active suspension offers tailor-made flexibility" (Press release). Audi. (2017-06-22). Retrieved (2017-06-24)

4. Aleksandr Grek. // Populyarnaya mechanika. — № 12 (38), (December 2005)

5. Connor, Jerome J. "Chapter 4: Tuned Mass Damper Systems" (PDF). Introduction to Structural Motion Control. Prentice Hall Pearson Education. p. 223. ISBN 9780130091383. (2003) 